\title{
CAUSALIDADE E TRANSMISSÃO ENTRE OS PREÇOS DE MANDIOCA, TRIGO, MILHO E SEUS DERIVADOS NO PARANÁ ${ }^{1}$
}

\author{
Lucilio Rogerio Aparecido Alves ${ }^{2}$ \\ Carlos Estevão Leite Cardoso ${ }^{3}$ \\ Fábio Isaias Felipe 4 \\ Michele Tubero Campion
}

\begin{abstract}
Resumo - O objetivo deste trabalho foi analisar as relações de causalidade e de transmissão de preços da raiz de mandioca, da fécula de mandioca, da farinha de mandioca, do trigo, da farinha de trigo e do milho, no Estado do Paraná. Os dados foram referentes ao período de janeiro de 1995 a dezembro de 2005, com periodicidade mensal. Utilizou-se a metodologia de causalidade de Granger para testar as relações entre as variáveis. Os resultados apontaram para relações de longo prazo entre milho e mandioca, fécula e farinha de mandioca, trigo e milho e trigo e farinha de trigo. Ressaltou-se a dependência dos preços da raiz de mandioca em relação aos preços do milho, do trigo, da fécula e da farinha de mandioca e sua pouca influência sobre essas variáveis. Destacou-se a influência dos preços da farinha de mandioca sobre os da fécula, assim como a forte relação entre os preços do trigo e da farinha de trigo. Os preços da farinha de mandioca e do trigo mostraram menor dependência das demais variáveis analisadas. Em suma, pode-se dizer que os resultados mostraram importantes inter-relações entre as variáveis. O preço da raiz de mandioca foi o que mostrou maior dependência das demais variáveis.

Palavras-chave: causalidade de Granger, transmissão de preços, amido.
\end{abstract}

Os autores são gratos aos pareceristas desta Revista pelas preciosas sugestões e comentários.

2 Pesquisador do Centro de Estudos Avançados em Economia Aplicada - CEPEA/ESALQ/USP. E-mail: lualves@esalq.usp.br

3 Pesquisador da Embrapa Mandioca e Fruticultura Tropical e pesquisador convidado do CEPEA/ESALQ/ USP.E-mail: estevao@cnpmf.embrapa.br

4 Pesquisador do CEPEA/ESALQ/USP. E-mail: fifelipe@esalq.usp.br

5 Estagiária do CEPEA/ESALQ/USP. E-mail: mcampion@esalq.usp.br 


\section{Introdução}

O objetivo deste trabalho foi, por meio de dados de periodicidade mensal, analisar as relações de causalidade entre os preços da raiz de mandioca, da fécula de mandioca, da farinha de mandioca, do trigo, da farinha de trigo e do milho, identificando-se o sentido e a transmissão de preços entre esses produtos, no Estado do Paraná. Os preços do amido de milho não foram considerados no presente estudo, devido à sua indisponibilidade. Os dados são referentes ao período de janeiro de 1995 a dezembro de 2005.

No Estado do Paraná, foco deste trabalho, há indicativos de forte concorrência por área para o plantio entre as culturas da mandioca e do milho, e do milho com a do trigo. A opção pelo plantio de mandioca pode implicar, pelo menos, exclusão de uma cultura de verão, soja ou milho, por exemplo, e de uma de inverno, milho ou trigo, entre outras. Há também forte disputa por mercados que utilizam amidos e farinha como insumo ou mesmo como um produto de consumo final.

Em especial, a cadeia agroindustrial da mandioca vem, nos últimos anos, passando por grandes mudanças decorrentes de alterações no padrão de consumo dos produtos, de modo geral, e da maior utilização do amido de mandioca, muitas vezes em substituição a outros amidos que apresentam preços mais elevados. No Brasil, as regiões Norte e Nordeste são as principais consumidoras de mandioca, sendo seu uso concentrado na alimentação humana, principalmente na forma de farinha. Nas regiões Sul, Sudeste e Centro-Oeste, a principal destinação das raízes é a indústria, e os pólos mais importantes de beneficiamento estão no Paraná, Mato Grosso do Sul, São Paulo e Santa Catarina. O Estado do Paraná é o maior produtor de fécula de mandioca do Brasil.

O milho tem grande importância econômica e social para o Estado do Paraná, uma vez que é grande empregadora de mão-de-obra. Estima-se que $60 \%$ da área cultivada no estado seja ocupada com a cultura do milho. O principal produtor nacional, em 2005, foi o Paraná, responsável 
Lucilio Rogerio Aparecido Alves, Carlos Estevão Leite Cardoso, Fábio Isaias Felipe \& Michele Tubero Campion

por $24,4 \%$ da produção. Em seguida vêm Minas Gerais e São Paulo, com $17,8 \%$ e $11,6 \%$, respectivamente.

A produção de trigo tem pequena participação na produção brasileira de grãos. Mesmo assim, seu derivado, a farinha, é importante para o setor alimentício brasileiro, principalmente através de produtos oriundos da panificação. Os principais estados produtores de trigo são Paraná e Rio Grande do Sul, responsáveis por 75,6\% da produção total nos últimos 10 anos. No ano de 2005, o Paraná foi responsável por 48,4\% e o Rio Grande do Sul por 27,2\% da quantidade produzida no Brasil.

É nesse contexto que este trabalho se insere. Buscar entender a interrelação e a causalidade que há entre os preços dos produtos agrícolas mandioca, milho e trigo, assim como a interação de cada um com a fécula e com as farinhas de mandioca e de trigo, parece ser uma importante informação, sobretudo para os atores das cadeias agroindustriais em estudo.

Nas análises de transmissão e causalidade que serão efetuadas neste trabalho, espera-se encontrar relação entre os preços da raiz de mandioca com os do milho e do trigo. Relações entre os preços da fécula e da farinha de mandioca com os da raiz de mandioca, e vice-versa, também serão estimadas. Também há a hipótese da existência de relação entre os preços da fécula com a farinha de mandioca e com a farinha de trigo. Para a farinha de trigo, por sua vez, espera-se encontrar maior relação com o preço do trigo do que com as demais variáveis do modelo. Com base nos testes estatísticos específicos, será possível identificar o sentido da causalidade entre as variáveis em estudo. Por exemplo, é interessante saber se é o preço da fécula influenciado pelo preço da matéria-prima ou se é o preço da fécula que influencia o preço da raiz de mandioca. Além disso, pouco se sabe sobre a interação entre os preços da fécula e os da farinha de trigo.

O presente estudo foi estruturado da seguinte forma. Além desta parte introdutória, em seguida se descreve a revisão bibliográfica sobre trabalhos 
que analisaram relações de preços entre produtos agrícolas e pecuários. A metodologia utilizada é apresentada na seção três. Na seção quatro, os resultados das relações estimadas são apresentados e discutidos. As considerações finais sumarizam este trabalho.

\section{Revisão de literatura}

Nesta seção, buscar-se-á efetuar uma revisão de literatura sobre relações de preços de produtos da agropecuária. As metodologias utilizadas também serão citadas.

Martines Filho (1988) analisou o sentido de causalidade de séries de preços agrícolas entre os níveis de produtor, atacado e varejo, bem como a elasticidade com que os preços são transmitidos entre cada um desses níveis de mercado e o comportamento das margens de comercialização. Utilizando dados para o período de 1972 a 1985, o autor trabalhou com as seguintes variáveis: arroz em casca, milho em grão, soja em grão, laranja de mesa, arroz sem casca, fubá mimoso e óleo de soja refinado enlatado.

Utilizando a metodologia de Granger, os resultados mostraram que na maioria dos casos em estudo os choques nos preços iniciaram-se no âmbito da produção de matéria-prima, provavelmente por razões climáticas. Para os produtos destinados ao mercado externo, acredita-se que as exportações podem desencadear inicialmente as variações de preços aos produtores.

Bliska (1989) analisou as relações de transmissão de preços entre os níveis de mercado de carne bovina numa única análise, estudando os mecanismos através dos quais se propagam os choques nesses preços, determinando a intensidade e a duração de tais choques e calculando as margens de comercialização. Nos testes de causalidade, a autora utilizou séries de preços médios mensais reais recebidos pelos mercados atacadista paulista, varejista da cidade de São Paulo e produtores de boi 
Lucilio Rogerio Aparecido Alves, Carlos Estevão Leite Cardoso, Fábio Isaias Felipe \& Michele Tubero Campion

gordo dos estados de São Paulo, Minas Gerais, Goiás, Mato Grosso e Rio Grande do Sul, no período de janeiro de 1971 a dezembro de 1987. Foi estimado pela autora um modelo de Auto-Regressão Vetorial (VAR), constituído das variáveis preços do boi gordo em nível de produtor, preços da carcaça no atacado e preços da carne bovina no varejo. Dessa forma, foi detectada causalidade instantânea entre as variáveis. Verificou-se que a intensidade dos efeitos de choques sobre os níveis de varejo e atacado decresce rapidamente, anulando todos os efeitos nos primeiros meses após um choque nos preços. Os choques nos preços de boi gordo persistem por períodos mais longos.

Guimarães (1990) estudou a formação e transmissão de preços no mercado de frango (produtor, atacado e varejo) no Estado de São Paulo, considerando também a interação com os mercados de carne bovina e de milho e o mercado externo de frango. Buscou-se, assim, analisar as relações entre os níveis de mercado quanto a alterações de preços e determinar a intensidade e a duração da propagação de choques entre tais níveis e entre o mercado de frango e os outros mercados considerados. A metodologia utilizada foi a Auto-Regressão Vetorial (VAR). A conclusão do autor foi de que o preço em São Paulo é informativo para os preços em níveis de atacado e varejo, sem relação de causalidade entre os dois últimos níveis. Quando se adiciona o preço ao produtor de Santa Catarina, os resultados indicam os preços ao atacado e varejo em São Paulo como informativos para o preço ao produtor catarinense.

De Zen (1997) buscou detectar as relações entre os preços diários de boi gordo nas diversas regiões do Centro-Sul do Brasil e entre esses preços e o da carne bovina na região da Grande São Paulo. Verificou-se, também, a existência de integração entre as diversas praças produtoras e entre os diferentes níveis de mercado.

Nesse sentido, vários modelos foram ajustados para detectar a existência de causalidade entre as séries de preços diários do boi gordo. Os resultados mostraram um mercado altamente integrado, com respostas rápidas para alterações de preços nas regiões produtoras. Observou-se ainda que 
alterações de preços podem ser causadas por fatores próprios de cada região e ser transmitidas para outras.

Aguiar (1990) analisou o processo de formação de preços em vários níveis de mercado na indústria brasileira de soja, no Estado de São Paulo. Considerou-se como centro de consumo para o óleo no atacado e no varejo a cidade de São Paulo, e a região de Campinas para o farelo, no atacado. Foi também considerado o mercado externo de soja e derivados, usando dados mensais para o período entre fevereiro de 1992 e novembro de 1989. Analisou-se o sentido da causalidade através do teste de GrangerSims e estimaram-se as elasticidades de transmissão de preços. Os resultados mostraram que a principal origem dos choques de preços é o mercado internacional. No mercado interno, o nível de preços no atacado tende a iniciar as variações de preços. Observou-se ainda que o único nível de mercado interno que afeta o mercado externo é o atacado de farelo, tendo, assim, uma relação bicausal.

Já Mafioletti (2001) analisou o processo de formação dos preços da soja tanto em níveis de produtor, atacado e varejo e mercado externo quanto entre as principais regiões produtoras e consumidoras de soja no mercado interno, entre 1992 e 1999. As regiões estudadas foram Rio Grande do Sul, Paraná, São Paulo, Goiás, Mato Grosso do Sul e Mato Grosso. A metodologia utilizada foi o teste de Granger. Chegou-se aos resultados de que o período máximo de transmissão de preços no setor é de um mês, e somente alguns casos apresentando dois ou três meses. Esses resultados indicaram que há eficiência na transmissão de preços entre os níveis de mercado e as regiões estudadas.

Ferreira (2001) estudou a produção e a comercialização do feijão considerando as épocas de colheita, a distribuição geográfica e os principais fluxos de distribuição. Também foram analisadas as relações de preços recebidos pelos produtores entre os preços ao atacado e varejo na cidade de São Paulo. 
Lucilio Rogerio Aparecido Alves, Carlos Estevão Leite Cardoso, Fábio Isaias Felipe \& Michele Tubero Campion

Os resultados indicaram que após o Plano Real ocorreu crescimento na produção na região Noroeste, mas sem alterar a produção nos tradicionais estados produtores. Do mesmo modo, observou-se aumento na importação. Portanto, há concentração da produção de feijão no Brasil, e a produção pulverizada desempenha papel importante no comportamento do mercado.

Souza e Stülp (2005) examinaram as relações de trocas e a causalidade de Granger entre preços pagos e recebidos pela agropecuária brasileira, entre 1986 e 2004. Os autores concluíram que os preços recebidos não causaram os preços pagos totais; contudo, causaram os preços pagos por combustíveis, sementes e serviços antes do Plano Real e por agrotóxicos, sementes e serviços após 1994. O efeito dos preços pagos sobre os recebidos foi maior no primeiro período do que no segundo. Com a abertura da economia, os preços agrícolas passaram a ser influenciados mais por fatores internacionais do que pelos preços internos dos insumos.

Margarido et al. (2006) analisaram a elasticidade de transmissão de preços entre os preços da farinha de trigo na cidade de São Paulo, da cotação internacional do grão de trigo e da taxa de câmbio, entre janeiro de 1999 e dezembro de 2005 . Foram utilizados vários métodos relacionados com séries de tempo, entre eles o de causalidade de Granger e o Modelo Vetorial de Correção de Erro. Os resultados mostram que, no longo prazo, variações das cotações internacionais do trigo em grão e da taxa de câmbio são plenamente transmitidas para os preços da farinha de trigo na cidade de São Paulo, corroborando uma hipótese de modelo de Lei do Preço Único nesse mercado.

Estudos que analisam relações de preços nos mercados de raiz e fécula de mandioca são raros. Melo Filho e Otsubo (1999) analisaram a estacionariedade e a tendência dos preços de mandioca no Estado do Mato Grosso do Sul, considerando dados de 1980 a 1998. Em termos de variação estacional, concluíram que os maiores preços ocorreram entre os meses de março e julho, seguido de um período de menores preços, 
entre os meses de agosto e dezembro. Também observaram que no período do estudo os preços apresentaram um movimento descendente ao longo do tempo, associado, entre outros motivos, ao aumento da oferta do produto.

Otsubo e Lorenzi (2002) analisaram a variação estacional dos preços de mandioca pagos pela indústria, em Mato Grosso do Sul, no período de 1998 a 2001. Os autores observaram que, dada a escassez do produto que normalmente ocorre, o período de preços mais altos ocorre entre os meses de outubro e março. No mês de fevereiro foi observado o maior preço. De abril a setembro os preços situam-se em patamares mais baixos, sendo essa a época de maior oferta do produto. No mês de junho observou-se o menor preço.

Silva et al. (2003) também analisaram a tendência de preços de mandioca para os estados de São Paulo e Paraná, no período de 1980 a 2001. Os autores estudaram a sazonalidade de preços nos dois estados, tanto no período total do estudo como em subperíodos. Para o Estado do Paraná, no subperíodo de 1980-1989, o maior índice sazonal ocorreu no mês de abril e o menor em julho. De 1990 a 2001 o maior índice ocorreu em janeiro. Quando se considera apenas o período pós-Plano Real, o maior índice desloca-se para o mês de dezembro, sugerindo uma antecipação do período de safra. Para o Estado de São Paulo, a única diferença em relação aos resultados obtidos para o Paraná refere-se ao primeiro subperíodo, que apresentou o maior índice no mês de janeiro. Também se observou uma tendência de preços decrescentes no período total sob análise.

Alves e Vedovoto (2003) estudaram a formação e transmissão, assim como as margens e as relações causais, de preços ao longo da cadeia de mandioca (produção de raiz, fécula e farinha), usando dados mensais e agregados para os estados do Paraná, Bahia e São Paulo. Os autores observaram que, em toda a região Centro-Sul do Brasil, os preços são menores entre os meses de maio e agosto, período em que é concentrada a oferta de mandioca. Em se tratando de margens, para o Estado do 
Lucilio Rogerio Aparecido Alves, Carlos Estevão Leite Cardoso, Fábio Isaias Felipe \& Michele Tubero Campion

Paraná a margem do atacadista de farinha foi decrescente de 1982 a 2001, enquanto a margem do varejista foi crescente. Para o Estado de São Paulo, as margens não apresentaram uma tendência definida. Em termos gerais, os preços da fécula no atacado acompanham as variações da raiz. O preço da farinha variou proporcionalmente menos do que os da raiz e da fécula. $\mathrm{Na}$ análise de causalidade, esses autores verificaram relações causais do preço da raiz do estado da Bahia para o Paraná, deste para São Paulo e de São Paulo para os estados da Bahia e do Paraná. As relações causais entre os mercados de fécula e de farinha nos três estados também foram analisadas. Esses resultados também foram comprovados por Barros et al. (2004).

Santana et al. (2005), ao analisarem as variações de preços de raiz de mandioca no Estado da Bahia entre 1990 e 2004, constataram sazonalidade nos preços recebidos pelos produtores de raiz de mandioca. Observaram que nos meses de maio a novembro ocorrem os menores preços, e de dezembro a março, os preços mais elevados.

Em seguida, descreve-se a metodologia que será utilizada neste trabalho para analisar as relações de causalidade e de transmissão de preços da raiz de mandioca, da fécula de mandioca, da farinha de mandioca, do trigo, da farinha de trigo e do milho, no Estado do Paraná.

\section{Referencial metodológico}

Os testes de causalidade realizados neste trabalho foram implementados utilizando a metodologia de Granger (1969), a qual foi adotada com a finalidade de analisar se os movimentos de preço de um mercado precedem os movimentos de preço de outro mercado. As seguintes equações compõem o teste de causalidade entre preços de dois mercados, podendo ser estimadas por Mínimos Quadrados Ordinários (Gujarati, 1995): 


$$
\begin{aligned}
& Y_{t}=\gamma+\sum_{i=0}^{n} \alpha_{i} X_{t-i}+\sum_{j=1}^{m} \beta_{j} Y_{t-j}+u_{1 t} \\
& X_{t}=\theta+\sum_{i=0}^{p} \rho_{i} Y_{t-i}+\sum_{j=1}^{q} \varpi_{j} X_{t-j}+u_{2 t}
\end{aligned}
$$

nas quais: $Y_{t}$ e $X_{t}$ são os preços de interesse; $\gamma, \alpha_{i}, \beta_{j}, \theta, \rho_{i}$ e $\varpi_{j}$ são os parâmetros a serem estimados; $i$ e $j$ são os números de defasagens dos preços; e $u_{i t}$ são os erros aleatórios não-correlacionados.

Causalidade entre preços existe se pelo menos um dos coeficientes da variável explicativa defasada for estatisticamente diferente de zero em uma ou nas duas equações. Três situações podem ocorrer: (i) relação bicausal, quando os efeitos ocorrem nas duas direções - nesse caso, o teste F é significativo para (1) e (2); (ii) ausência de causalidade - o teste $\mathrm{F}$ apresenta-se não-significativo para as duas equações; e (iii) relação unicausal, quando o efeito ocorre numa única direção - o teste F de uma das equações é estatisticamente significativo e o da outra não. Nesse último caso, o sentido de causalidade é da variável explicativa para a dependente, considerando a equação em que o teste $\mathrm{F}$ apresentou-se significativo.

As elasticidades de transmissão de preço são obtidas por meio dos coeficientes das variáveis explicativas, sendo os próprios coeficientes se os dados forem transformados em logaritmos para o ajustamento das equações.

Se as variáveis não forem estacionárias em nível, os modelos deverão ser ajustados com as séries nas diferenças; se as séries forem cointegradas, os modelos deverão incluir um termo de correção de erro. As metodologias de Fuller (1976) e Dickey e Fuller (1979 e 1981) foram 
Lucilio Rogerio Aparecido Alves, Carlos Estevão Leite Cardoso,

Fábio Isaias Felipe \& Michele Tubero Campion

utilizadas para testar a estacionariedade das séries, e a metodologia de Engle e Granger (1987), para testar co-integração entre as variáveis. ${ }^{6}$

\section{Resultados e discussão}

Antes de analisar os resultados da inter-relação entre os preços da mandioca, do milho e do trigo e seus derivados, apresentar-se-á a evolução das séries de preços utilizadas. O preço da fécula foi aquele pago para retirar o produto na indústria, assim como os preços das farinhas de mandioca e de trigo, ambos em nível de indústria.

\subsection{Dados utilizados}

Os dados de preços de raiz de mandioca, de milho e de trigo, recebidos pelo produtor no Estado do Paraná, foram obtidos na base da Fundação Getúlio Vargas (FGV). Os dados de preços de fécula de mandioca, da farinha de mandioca crua e da farinha de trigo foram da Secretaria de Agricultura e Abastecimento do Paraná, os quais são levantados pelo Departamento de Economia Rural (Seab/Deral). As séries foram deflacionadas pelo Índice Geral de Preços - Disponibilidade Interna (IGPDI), da Fundação Getúlio Vargas (FGV), base dez./05 = 1,00.

A evolução dos preços da mandioca, do trigo e do milho pode ser analisada na Figura 1. Em termos gerais, observa-se a expressiva oscilação dos preços, principalmente os da mandioca. Até o ano de 2002, as séries apresentam comportamentos semelhantes. Entretanto, o preço da raiz de mandioca apresentou maior crescimento, comparativamente aos demais, no período entre o segundo semestre de 1996 e o primeiro semestre de 2001. Em seguida, os preços da mandioca decresceram, enquanto os demais tiveram um pico de valorização. Todavia, a partir de 2003, com a

Informações sobre testes de raiz unitária e de co-integração de Engle e Granger podem ser obtidas, entre outros trabalho, em: Lütkepohl (1991), Gujarati (1995), Enders (1995), Bacchi (1994), Bittencourt (1995) e Alves (2002). 
escassez de matéria-prima, os preços da mandioca cresceram expressivamente, chegando a ser 3,25 vezes maior que o observado em janeiro de 1995. Essa valorização se estendeu até o início do ano de 2004, quando voltou a apresentar decréscimos. Numa análise geral, podese dizer que os preços observados atualmente, para os três produtos em estudo, estão próximos dos patamares obtidos entre os anos de 1997 e 1999. Entretanto, os preços dos produtos derivados mostraram maiores oscilações que os da matéria-prima, mas com tendência semelhante.

A farinha de trigo praticamente reproduziu o comportamento dos preços do trigo, apontando para uma importante interdependência (Figura 2). Já os preços da fécula e da farinha de mandioca crua, além de não acompanharem a valorização dos preços da raiz de mandioca, na maioria dos períodos, tiveram decréscimos, com exceção do período em que os preços da raiz valorizaram além do padrão normal. Atualmente, os preços reais destes produtos estão próximos dos observados entre os anos de 2001 e 2002, que foram os anos de menores preços. Embora esse comportamento dos preços represente perda de renda por parte dos agricultores e das indústrias de fécula, por outro lado, indica maior poder concorrencial em relação aos produtos substitutos, como o amido de milho e a farinha de trigo. 
Lucilio Rogerio Aparecido Alves, Carlos Estevão Leite Cardoso,

Fábio Isaias Felipe \& Michele Tubero Campion

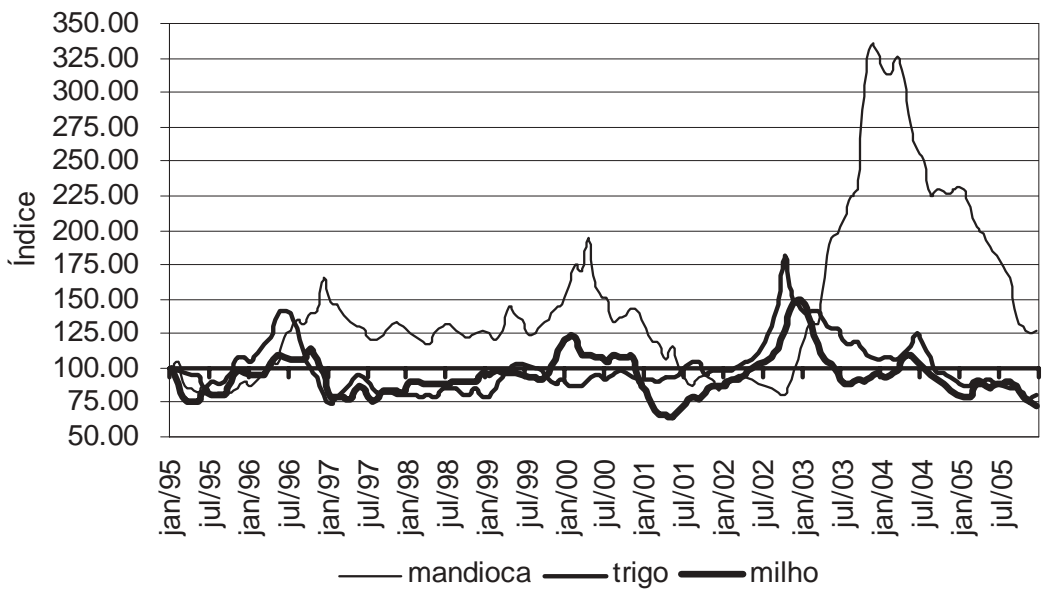

Figura 1 - Evolução das séries de preços da raiz de mandioca, do trigo e do milho, em número- índice - jan./1995 a dez./2005.

Fonte: Fundação Getúlio Vargas - FGV (2006).

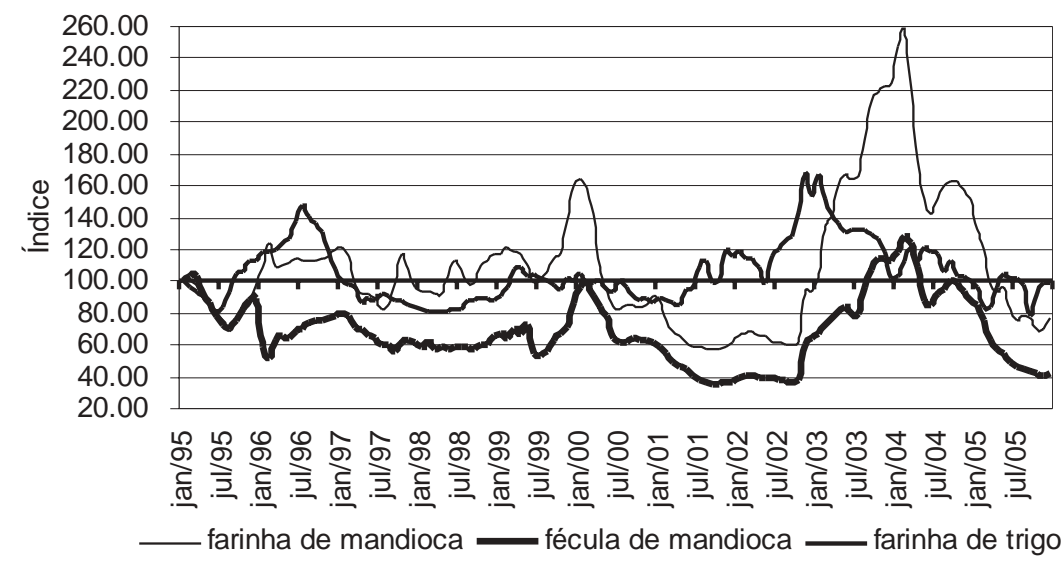

Figura 2 - Evolução das séries de preços da farinha de mandioca crua, da fécula e da farinha de trigo, em número-índice - jan./1995 a dez./2005.

Fonte: Secretaria de Estado da Agricultura e do Abastecimento do Paraná Seab (2006). 
4.2. Propriedades de raiz unitária e de co-integração das variáveis

Os resultados dos testes de raiz unitária das séries de preços constam na Tabela 1. Inicialmente, os testes de Akaike e Schwarz sugerem um modelo auto-regressivo de ordem seis [AR(6)] para a variável preço da raiz de mandioca e de ordens menores [AR(2) e AR(1)] para as demais variáveis (segunda coluna da Tabela 1). Esses resultados indicam que todas as séries de preços sofrem influência dos preços passados na formação dos preços atuais, e isso se refere aos preços de um mês anterior a até seis meses anteriores. Na realização do teste de Dickey-Fuller Aumentado (ADF), adotou-se o procedimento proposto por Enders (1995) para identificar o modelo específico a ser considerado, utilizando o sistema sequiencial descrito anteriormente.

Os resultados dos testes de ADF indicam que todas as séries são integradas de ordem um [I(1)] no modelo sem termos deterministas (modelo 1). Repetiu-se o ajustamento para verificar se as séries tornamse estacionárias quando analisadas nas primeiras diferenças. Esses resultados são apresentados na última coluna da Tabela 1 (modelo 2), onde se verifica que os coeficientes são significativos. 
Lucilio Rogerio Aparecido Alves, Carlos Estevão Leite Cardoso, Fábio Isaias Felipe \& Michele Tubero Campion

Tabela 1 - Resultados dos testes de raiz unitária de Dickey-Fuller para as séries de preços de raiz de mandioca, de milho, de trigo, de fécula de mandioca, de farinha de mandioca e de farinha de mandioca crua - Estado do Paraná

\begin{tabular}{lccccccc}
\hline \multicolumn{1}{c}{ Variáveis } & Valor & \multicolumn{5}{c}{ Modelo 1* } & \multicolumn{3}{c}{ Modelo 2** } \\
\cline { 2 - 7 } & de $p-1$ & $\tau_{\tau}$ & $\tau_{\beta \tau}$ & $\tau_{\mu}$ & $\tau_{\alpha \mu}$ & $\tau$ & $\tau$ \\
\hline Mandioca & 5 & $-2,785$ & 0,913 & $-2,781$ & 2,781 & $-0,107$ & $-2,952^{\#}$ \\
Trigo & 1 & $-2,579$ & 0,317 & $-2,600$ & 2,591 & $-0,305$ & $-7,748^{\#}$ \\
Milho & 1 & $-3,488$ & $-0,132$ & $-3,558^{\#}$ & $3,553^{\#}$ & $-0,197$ & $-6,965^{\#}$ \\
Fécula & 1 & $-2,381$ & $-0,114$ & $-2,388$ & 2,334 & $-0,814$ & $-8,097^{\#}$ \\
Far. crua mand. & 1 & $-2,172$ & $-0,023$ & $-2,240$ & 2,215 & $-0,416$ & $-7,106^{\#}$ \\
Far.trigo & 0 & $-2,083$ & 0,136 & $-2,122$ & 2,117 & $-0,190$ & $-10,271^{\#}$ \\
\hline
\end{tabular}

\# Significativo a 1\% de significância.

* Modelo $1 \Rightarrow \Delta x_{t}=\alpha+\beta t+\gamma x_{t-1}+\sum_{i=1}^{p-1} \lambda_{i} \Delta x_{t-i}+\varepsilon_{t}$, nas versões com constante e tendência, sem tendência e sem tendência e constante.

** Modelo $2 \Rightarrow \Delta \Delta x_{t}=\gamma \Delta x_{t-1}+\sum_{i=1}^{p-2} \lambda_{i} \Delta \Delta x_{t-i}+\varepsilon_{t}$, definido após constatada a não-existência de termos deterministas.

Obs.: não houve presença de autocorrelação serial, conforme o teste $Q$ de Ljung Box.

Fonte: Dados da pesquisa.

A variável preço do milho sinalizou estacionariedade em nível, mas, devido à proximidade entre os valores calculados e os da tabela de valores críticos, optou-se por utilizá-la na primeira diferença. ${ }^{7}$ Dessa forma, as variáveis são consideradas como integradas de ordem um [I(1)] e, na especificação do modelo para o teste de causalidade, devem ser consideradas nas diferenças de primeira ordem.

Em seguida, efetuou-se o teste de co-integração de Engle e Granger (1987) para analisar as relações de longo prazo entre as séries. Os

\footnotetext{
Observa-se, entretanto, que, se fosse utilizado um nível de significância de 5\%, os preços da mandioca e do trigo seriam também séries estacionárias em nível, segundo o procedimento de Enders.
} 
resultados são mostrados na Tabela 2, onde já se apresentam todas as causalidades que foram testadas neste trabalho. Testou-se a causalidade dos preços da mandioca com os do trigo, do milho, da farinha de mandioca crua e da fécula. Para os preços da fécula, analisaram-se as relações com preços da mandioca, da farinha de mandioca crua e da farinha de trigo. A causalidade dos preços da farinha de mandioca crua foi testada com o preço da farinha de trigo, além dos preços da mandioca e da fécula. A causalidade entre os preços do milho com os do trigo e da mandioca também foi analisada. Já o preço do trigo foi testado em relação ao da mandioca, do milho e da farinha de trigo. Por fim, a relação dos preços da farinha de trigo com os do trigo, da farinha de mandioca e da fécula foi analisada. 
Lucilio Rogerio Aparecido Alves, Carlos Estevão Leite Cardoso,

Fábio Isaias Felipe \& Michele Tubero Campion

Tabela 2 - Resultados dos testes de co-integração de Engle e Granger para as séries de preços de raiz de mandioca, de milho, de trigo, de fécula de mandioca, de farinha de mandioca e de farinha de mandioca crua - Estado do Paraná

\begin{tabular}{cccl}
\hline Variável dependente & Variável explicativa & Valor de $m$ & Valor calculado de $\tau$ \\
\hline Mandioca & Trigo & 5 & $-2,797$ \\
Trigo & Mandioca & 1 & $-2,609$ \\
Mandioca & Milho & 5 & $-2,777$ \\
Milho & Mandioca & 1 & $-3,578^{\# \#}$ \\
Mandioca & Far. Mand. Crua & 0 & $-2,351$ \\
Far. Mand. Crua & Mandioca & 1 & $-2,770$ \\
Mandioca & Fécula & 0 & $-2,208$ \\
Fécula & Mandioca & 1 & $-2,710$ \\
Fécula & Far. Mand. Crua & 1 & $-3,908^{\#}$ \\
Far. Mand. Crua & Fécula & 1 & $-3,678^{\#}$ \\
Trigo & Milho & 1 & $-3,110^{\# \#}$ \\
Milho & Trigo & 1 & $-3,874^{\#}$ \\
Trigo & Far. Trigo & 0 & $-5,002^{\#}$ \\
Far. Trigo & Trigo & 0 & $-5,216^{\#}$ \\
Far. Trigo & Far. Mand. Crua & 0 & $-2,232$ \\
Far. Mand. Crua & Far. Trigo & 1 & $-2,322$ \\
Far. Trigo & Fécula & 0 & $-2,166$ \\
Fécula & Far. Trigo & 1 & $-2,422$ \\
\hline
\end{tabular}

\# Significativo a 1\% de significância; \#\# Significativo a 5\% de significância [valores críticos em Engle e Granger (1987)].

Obs.: Modelo utilizado $\Rightarrow \Delta \varepsilon_{t}=\pi \varepsilon_{t-1}-\sum_{j=1}^{m} \omega_{j} \Delta \varepsilon_{t-j}+u_{t}$.

Não houve presença de autocorrelação serial, conforme o teste $Q$ de Ljung Box.

Fonte: Dados da pesquisa.

O número de defasagens utilizado no teste de co-integração foi determinado de acordo com os critérios de Akaike e Schwarz, os quais indicaram que os resíduos são descritos por um processo que oscila entre um modelo $\mathrm{AR}(1)$ e um $\mathrm{AR}(6)$. A aplicação do teste de ADF para os resíduos (Tabela 2) aponta para a relação de longo prazo entre as seguintes variáveis: preços do milho e da mandioca, no modelo em que se testa a 
causalidade da primeira variável sobre a segunda; preço da fécula com o da farinha de mandioca crua; preço do milho com o do trigo; e preço do trigo com o da farinha de trigo. Dessa forma, a maioria das relações não foi estatisticamente significativa, como aquelas entre os preços da mandioca e da fécula. Contudo, esse resultado reflete as tendências distintas que tiveram as duas séries ao longo do período analisado, como demonstrado nas Figuras 1 e 2.

Nas relações em que o modelo sugere co-integração das séries, ou seja, relações de equilíbrio de longo prazo, o modelo de causalidade ajustado foi, portanto, com Correção de Erro. Assim, os modelos passaram a incorporar os aspectos tanto de curto quanto de longo prazos.

Na Tabela 3 apresentam-se os resultados dos testes de causalidade entre os preços descritos anteriormente. Para definição do número de defasagens da variável explicativa nos modelos ajustados para a análise de causalidade e transmissão de preços, foi construída uma função de correlação cruzada com as séries nas diferenças. Numa segunda etapa, foi identificado, após ajustar o modelo com as defasagens da variável explicativa, o número de termos auto-regressivos necessários para que o resíduo se apresente não- correlacionado.

Em seguida, serão analisadas as relações de causalidade e de elasticidade de transmissão de preços. Para definição do número de defasagens da variável explicativa nos modelos ajustados, foi construída uma função de correlação cruzada com as séries nas diferenças. Numa segunda etapa, foi identificado, após ajustar o modelo com as defasagens da variável explicativa, o número de termos auto-regressivos necessários para que o resíduo se apresente não-correlacionado. 


\subsection{Testes de causalidade de Granger}

Na Tabela 3, os resultados mostram que os preços da raiz de mandioca sofrem influência dos preços do trigo, do milho, da fécula e da farinha de mandioca crua. Assim, oscilações de preços dessas variáveis causam impacto no preço da raiz de mandioca.

O interessante é que há relações de bicausalidade somente entre os preços de mandioca e de milho. Mais especificamente, os preços da raiz de mandioca sofrem influência daqueles supracitados, porém variações de preços da mandioca causam impactos somente no preço do milho, não tendo interferência sobre os preços de trigo, de farinha de mandioca e de fécula. 
Tabela 3 - Resultados dos testes de causalidade entre as séries de preços da raiz de mandioca, do milho, do trigo, da fécula de mandioca, da farinha de mandioca e da farinha de mandioca crua Estado do Paraná

\begin{tabular}{llc}
\hline \multicolumn{1}{c}{ De } & \multicolumn{1}{c}{ Para } & Nível de significância \\
\hline Trigo & Mandioca & 0,0014 \\
Milho & Mandioca & 0,0810 \\
Farinha de mandioca & Mandioca & 0,0012 \\
Fécula & Mandioca & 0,0009 \\
Mandioca & Fécula & 0,1310 \\
Farinha mandioca & Fécula & 0,0317 \\
Farinha de trigo & Fécula & 0,2248 \\
Mandioca & Farinha de mandioca & 0,4406 \\
Fécula & Farinha de mandioca & 0,1721 \\
Farinha de trigo & Farinha de mandioca & 0,0142 \\
Mandioca & Milho & 0,0849 \\
Trigo & Milho & 0,0808 \\
Mandioca & Trigo & 0,7532 \\
Milho & Trigo & 0,4890 \\
Farinha de trigo & Trigo & 0,0000 \\
Trigo & Farinha de trigo & 0,0393 \\
Farinha de mandioca & Farinha de trigo & 0,0975 \\
Fécula & Farinha de trigo & 0,1972 \\
\hline
\end{tabular}

Obs.: não houve presença de autocorrelação serial, conforme o teste $Q$ de Ljung Box.

Fonte: Dados da pesquisa.

Dessa forma, vale ressaltar a observação de que os preços da raiz de mandioca parecem ser determinados pelo comportamento dos preços dos produtos finais. Não é o preço da matéria-prima que irá determinar qual deverá ser o valor dos produtos finais, mas o contrário. Isso indica que o setor industrial tende a determinar o preço de venda dos produtos finais e, a partir do nível de markup, que considera adequado, encontra qual deverá ser o valor de compra e/ou o valor pago ao produtor da matéria-prima. 
Lucilio Rogerio Aparecido Alves, Carlos Estevão Leite Cardoso, Fábio Isaias Felipe \& Michele Tubero Campion

Salienta-se também que o preço da farinha de mandioca, no Estado do Paraná, sofre influência direta do preço desse produto na região Nordeste. Isso reforça a constatação de ausência de causalidade entre os preços da matéria-prima e dos produtos finais.

No caso da fécula de mandioca, as estatísticas também apontaram para resultados interessantes: houve não-significância estatística da causalidade dos preços da raiz sobre os da fécula; variações nos preços da farinha de mandioca causam impacto sobre os da fécula; e os preços da farinha de trigo não influenciam os da fécula, como esperado.

Dentre esses resultados, destaca-se a relação de causalidade dos preços da farinha de mandioca com os da fécula. Isso pode ser explicado pelo fato de que altas dos preços da farinha tendem a elevar a demanda e, conseqüentemente, os preços da matéria-prima, no intuito de aumentar a oferta de farinha. Como no Estado do Paraná os setores de farinha e fécula concorrem pela matéria-prima, acréscimos da demanda das farinheiras tendem a puxar, para cima, os preços da raiz. Se isso ocorre, o setor de fécula também deverá fazer o mesmo, na tentativa de estabilizar a entrega de matéria-prima por parte do produto. Com isso, provavelmente os preços da fécula se elevarão, mantendo estável a renda do setor industrial. Entretanto, esse é um aspecto que precisa de mais atenção em estudos posteriores. Cardoso (2003) já havia comentado a respeito da inter-relação entre os preços de farinha mandioca e de fécula.

Os preços da farinha de mandioca, por sua vez, apresentaram menor dependência das demais variáveis, considerando-se os resultados deste trabalho. Somente variações nos preços da farinha de trigo causam alterações nos da farinha de mandioca. Oscilações nos preços da raiz de mandioca e de fécula não impactam os da farinha de mandioca.

O preço do milho, por sua vez, sofre influência do preço de raiz de mandioca e de trigo. Esse resultado já era esperado e é importante, principalmente ao se analisar a possibilidade de substituição de áreas entre milho e trigo nas lavouras de "inverno", no Paraná. 
Quanto aos preços do trigo, estes sofrem influência dos preços da farinha de trigo, não havendo causalidade dos preços da raiz da mandioca e do milho para os do trigo. Esse resultado também era esperado para o caso da relação entre os preços do trigo e da raiz da mandioca. Assim, como no Brasil a maior parte do trigo é usada na forma de farinha, é a demanda deste setor que impacta seus preços. Entretanto, outros fatores, não relacionados neste trabalho, como os preços externos, devem influenciar os preços do trigo.

Por fim, observa-se que os preços da farinha de mandioca e os do trigo causam os preços da farinha de trigo. Como esperado, não se observou relação de causalidade do preço da fécula para o preço da farinha de trigo.

Bicausalidade foi observada apenas entre os preços da raiz de mandioca e os do milho, entre os preços da farinha de trigo e da farinha de mandioca e entre os preços do trigo e os da farinha de trigo. Por sua vez, vale ressaltar a ausência de causalidade entre os preços da farinha de trigo e os da fécula.

\subsection{Elasticidade de transmissão de preços}

Mesmo nas situações em que não houve presença de causalidade, algumas variáveis mostraram-se significativas quando se analisaram as elasticidades de transmissão de preços (Tabela 4). Os preços da raiz da mandioca respondem rapidamente às variações de preços do trigo, da farinha de mandioca e da fécula, com elasticidades contemporâneas de $-0,2826,0,1590$ e 0,2815 , respectivamente. Observa-se relação inversa entre os preços da raiz da mandioca e do trigo, enquanto os preços da raiz tendem a variar no mesmo sentido dos preços da fécula e da farinha de mandioca, conforme esperado. Nesse último caso, preços favoráveis para os produtos finais tendem a elevar o valor pago pela matéria-prima, na tentativa de aumentar o processamento e, conseqüentemente, a oferta de fécula e/ou farinha. 
Lucilio Rogerio Aparecido Alves, Carlos Estevão Leite Cardoso, Fábio Isaias Felipe \& Michele Tubero Campion

Os preços da raiz também respondem a variações nos preços da farinha de mandioca e da fécula, com uma defasagem. Os preços da fécula, especialmente, impactam os preços da raiz de mandioca com até três períodos de defasagem. Os preços do milho tendem a impactar os preços da raiz com duas defasagens, enquanto a influência do preço do trigo para o da raiz ocorre com sete defasagens. Portanto, variações nos preços dos produtos finais são repassadas mais rapidamente aos preços da raiz, ao passo que é preciso maior período para que estes absorvam variações de preços nos setores de milho e de trigo.

Variações nos preços de raiz e de farinha de mandioca impactam contemporaneamente os preços da fécula, e estes também respondem com duas defasagens às oscilações nos preços da raiz e da farinha de mandioca.

Os preços da farinha de mandioca respondem contemporaneamente a variações nos preços da raiz e da fécula, apesar de não ter sido observada a causalidade desses preços. Os preços da farinha de mandioca respondem com seis defasagens às variações nos preços da raiz. Apesar de haver causalidade entre os preços de farinha de trigo e de farinha de mandioca, a resposta ocorre com cinco defasagens.

Essas últimas considerações mostram a expressiva inter-relação entre os preços da raiz, da fécula e da farinha de mandioca, mesmo com a não-presença de causalidade em algumas das relações. Todavia, os preços da raiz e da fécula tendem a absorver com maior intensidade as variações de outras variáveis, comparativamente aos preços da farinha de mandioca. 
Tabela 4 - Elasticidades de transmissão de preços entre as séries de preços da raiz de mandioca, do milho, do trigo, da fécula de mandioca, da farinha de mandioca e da farinha de mandioca crua - Estado do Paraná

\begin{tabular}{|c|c|c|c|c|}
\hline De & Para & Contemporânea & Defasada & $\begin{array}{c}\text { Período de } \\
\text { defasagem (meses) }\end{array}$ \\
\hline Trigo & Mandioca & $-0.2826^{\# \#}$ & $0.4614^{\#}$ & 7 \\
\hline Milho & Mandioca & 0.0700 & $0.2857^{\# \#}$ & 2 \\
\hline Farinha mand. & Mandioca & $0.1590^{\# \#}$ & $0.2413^{\#}$ & 1 \\
\hline Fécula & Mandioca & $0.2815^{\#}$ & $\begin{array}{l}0.1482^{\# \#} \\
0.1776^{\# \#} \\
0.1492^{\# \#}\end{array}$ & $\begin{array}{l}1 \\
2 \\
3\end{array}$ \\
\hline Mandioca & Fécula & $0.4113^{\#}$ & $-0.1808^{\# \# \#}$ & 2 \\
\hline Farinha mand. & Fécula & $0.5505^{\#}$ & $0.1997^{\# \#}$ & 2 \\
\hline Farinha de trigo & Fécula & 0.1532 & 0.1490 & 1 \\
\hline Mandioca & Farinha mand. & $0.3086^{\#}$ & $-0.2069^{\# \# \#}$ & 6 \\
\hline Fécula & Farinha mand. & $0.5226^{\#}$ & -0.1151 & 6 \\
\hline Farinha de trigo & Farinha mand. & 0.1772 & $0.2706^{\# \#}$ & 5 \\
\hline Mandioca & Milho & 0.0270 & $-0.1343^{\# \#}$ & 3 \\
\hline Trigo & Milho & $0.1564^{\# \# \#}$ & $0.1716^{\# \#}$ & 3 \\
\hline Mandioca & Trigo & $-0.1870^{\#}$ & $-0.1264^{\# \# \#}$ & 10 \\
\hline Milho & Trigo & $0.1533^{\# \# \#}$ & 0.0623 & 1 \\
\hline Farinha de trigo & Trigo & $0.3249^{\#}$ & $0.2625^{\#}$ & 3 \\
\hline Trigo & Farinha trigo & $0.1326^{\# \# \#}$ & $-0.1894^{\#}$ & 5 \\
\hline Farinha mand. & Farinha trigo & 0.0959 & $-0.1151^{\# \# \#}$ & 1 \\
\hline Fécula & Farinha trigo & 0.0867 & $-0.1134^{\# \# \#}$ & 4 \\
\hline
\end{tabular}

\# Significativo a 1\% de significância; ${ }^{\#}$ Significativo a 5\% de significância; \#\#" Significativo a 10\% de significância.

Obs.: não houve presença de autocorrelação serial, conforme o teste $Q$ de Ljung Box.

Fonte: Dados da pesquisa.

Os preços do milho apresentaram influência contemporânea dos preços do trigo. Mesmo observando-se causalidade do preço da mandioca para o preço do milho, os impactos ocorrem com três defasagens. Nesse caso, o período de defasagem é maior que o observado na causalidade do preço do milho para o da mandioca. 
Lucilio Rogerio Aparecido Alves, Carlos Estevão Leite Cardoso, Fábio Isaias Felipe \& Michele Tubero Campion

Os preços do trigo sofrem influência contemporânea de variações nos preços da mandioca, do milho e da farinha de trigo. O preço da farinha de trigo causa impacto sobre o preço do trigo com uma defasagem. Apesar de não haver causalidade entre o preço da mandioca e do trigo, os resultados mostraram que, além da relação contemporânea, o preço da mandioca impacta o preço do trigo com dez defasagens.

Por último, os preços da farinha de trigo respondem contemporaneamente somente aos preços do trigo, enquanto variações nos preços da farinha de mandioca e da fécula tendem a influenciá-lo com uma e quatro defasagens, respectivamente. O preço da farinha de trigo também responde com cinco defasagens às variações no preço do trigo.

\section{Considerações finais}

Os resultados indicam relações de longo prazo somente entre os preços do milho e da mandioca, da fécula e da farinha de mandioca, do trigo e do milho e do trigo e da farinha de trigo. Dessa forma, chama a atenção a ausência de co-integração entre os preços da raiz de mandioca e seus derivados (fécula e farinha), bem como a presença de co-integração entre os preços do trigo e do milho, culturas concorrentes em área no Paraná, no plantio de inverno.

Nos testes de causalidade, ressalta-se a dependência dos preços da raiz de mandioca em relação aos preços do milho, do trigo, da fécula e da farinha de mandioca e sua pouca influência sobre estas variáveis. Entretanto, houve bicausalidade com os preços do milho e do trigo. Os preços da raiz de mandioca também respondem com defasagens às variações nos preços das demais variáveis.

Os preços da raiz de mandioca parecem ser determinados após a definição dos preços dos produtos finais e do markup do setor industrial. Ao mesmo tempo, a disputa por matéria-prima entre os setores de farinha e de fécula favorece variações de preços destes produtos derivados e uma inter-relação entre estes. 
Entre os demais resultados, destaca-se a influência dos preços da farinha de mandioca sobre os da fécula, assim como a forte relação entre os preços do trigo e da farinha de trigo. Os preços da farinha de mandioca e do trigo mostraram menor dependência das demais variáveis analisadas neste trabalho. No caso da farinha de mandioca, fatores relacionados à demanda interna e à concorrência com o produto de outras regiões produtoras devem ser mais relevantes para explicar suas variações. Para o trigo, os preços devem apresentar maior dependência da paridade de importação, dado o volume considerável de trigo importado pelo Brasil, principalmente da Argentina.

Em suma, pode-se dizer que os resultados mostraram importantes interrelações entre as variáveis estudadas. $\mathrm{O}$ preço da raiz de mandioca foi o que mostrou maior dependência das demais variáveis. Contudo, algumas relações devem fazer parte de novos trabalhos, entre elas a relação entre os preços da fécula e da farinha de mandioca, que se mostrou maior do que o esperado.

\section{Referências}

AGUIAR, D. R.D. de Formação de preços na industria brasileira de soja - 1982/1989. 1990. 140 p. Dissertação (Mestrado em Economia Aplicada) - Escola Superior de Agricultura "Luiz de Queiroz", Universidade de São Paulo, Piracicaba, 1990.

ALVES, E.R. de A.; VEDOVOTO, G.L. (Ed. Tec.). A indústria de amido de mandioca. Brasília, DF : Embrapa Informação Tecnológica, 2003.201p.

ALVES, L.R.A. Transmissão de preços entre produtos do setor sucroalcooleiro do Estado de São Paulo. 2002. 107 p. Dissertação (Mestrado em Economia Aplicada) - Escola Superior de Agricultura "Luiz de Queiroz", Universidade de São Paulo, Piracicaba, 2002. 
Lucilio Rogerio Aparecido Alves, Carlos Estevão Leite Cardoso, Fábio Isaias Felipe \& Michele Tubero Campion

BACCHI, M.R.P. Previsão de preços de bovino, suíno e frango com modelos de séries temporais. Piracicaba, 1994. 172 p. Dissertação (Mestrado em Economia Aplicada) - Escola Superior de Agricultura "Luiz de Queiroz", Universidade de São Paulo, Piracicaba, 1994.

BARROS, G.S. de C. (Coord.) Melhoria da competitividade da cadeia da mandioca no Estado de São Paulo. São Paulo : SEBRAE; Piracicaba : ESALQ : CEPEA, 2004.

BITTENCOURT, M.V.L. Formação de preços e caracterização do mercado de frango em São Paulo. Piracicaba, 1995. 161p. Dissertação (Mestrado em Economia Aplicada) - Escola Superior de Agricultura “Luiz de Queiroz”, Universidade de São Paulo.

BLISKA, F.M.M. Transmissão de preços de carne bovina entre níveis de mercado: uma aplicação do modelo de auto-regressão vetorial. Piracicaba, 1989. 209 p. Dissertação (Mestrado em Economia Aplicada) - Escola Superior de Agricultura "Luiz de Queiroz", Universidade de São Paulo, Piracicaba, 1989.

CARDOSO, C.E.L. Competitividade e inovação tecnológica na cadeia agroindustrial de fécula de mandioca no Brasil. Piracicaba, 2003. 188 p. Tese (Doutorado em Economia Aplicada) - Escola Superior de Agricultura "Luiz de Queiroz", Universidade de São Paulo, Piracicaba, 2003.

DE ZEN, S. Integração entre os mercados de boi gordo e de carne bovina nas regiões centro Oeste e Sudeste do Brasil. Piracicaba, 1987. 81 p. Dissertação (Mestrado em Economia Aplicada) - Escola Superior de Agricultura "Luiz de Queiroz", Universidade de São Paulo, Piracicaba, 1987.

DICKEY, D.A.; FULLER, W.A. Distribution of the estimator for autoregressive time series with a unit root. Journal of the American Statistical Association, v.74, n.366, p.427-431, 1979. 
DICKEY, D.A; FULLER, W.A. Likelihood ratio statistics for autoregressive time series with a unit root. Econometrica, v.49, n.4, p.10571072, jul. 1981.

ENDERS, W. Applied econometric time series. New York: John Wiley \& Sons, 1995. 433p.

ENGLE, R.F.; GRANGER, C.W.J. Co-integração and error corretion: representation, estimation, and testing. Econometrica, Chicago, v. 55, n. 2, p. 251-276, Mar. 1987.

FERREIRA, C.M. Comercialização de feijão no Brasil 1990-99. Piracicaba, 2001. 145 p. Dissertação (Mestrado em Economia Aplicada) - Escola Superior de Agricultura "Luiz de Queiroz", Universidade de São Paulo, Piracicaba, 2001.

FULLER, W.A. Introduction to statistical time series. 2.ed. New York: John Wiley \& Sons, 1976. 698p.

FUNDAÇÃO GETÚLIO VARGAS - FGV. FGVDADOS. Disponível em: 〈http://www2.fgv.br/dgd/asp/index.asp>. Acesso em: 10 fev. 2006.

GRANGER, C.W.J. Investigating causal relations by econometric models and cross spectral methods. Econometrica, v.37, n.3, p.424-38, July, 1969.

GUIMARÃES, V.D.A. Comercialização de transmissão de precos de carne de frango no estado de São Paulo. Piracicaba, 1990. 119 p. Dissertação (Mestrado em Economia Aplicada) - Escola Superior de Agricultura "Luiz de Queiroz", Universidade de São Paulo, Piracicaba, 1990.

GUJARATI, D.N. Basic econometrics. 3.ed. New York: McGrawHill, 1995. 838p. 
Lucilio Rogerio Aparecido Alves, Carlos Estevão Leite Cardoso, Fábio Isaias Felipe \& Michele Tubero Campion

INSTITUTO BRASILEIRO DE GEOGRAFIA E ESTATÍSTICA IBGE. Sistema IBGE de recuperação automática - SIDRA. Produção Agrícola Municipal. Disponível em: 〈http://www.sidra.ibge.gov.br〉. Acesso em: 10 fev. 2006.

LÜTKEPOHL, H. Introduction to multiple time series analysis. Berlin: Springer-Verlag, 1991. 764 p.

MAFIOLETTI, R.L. Formação de preços na cadeia agroindustrial da soja na década de 90. Piracicaba, 2001. 95 p. Dissertação (Mestrado em Economia Aplicada) - Escola Superior de Agricultura "Luiz de Queiroz”, Universidade de São Paulo, Piracicaba, 2001.

MARGARIDO, M.A.; BUENO, C.R.F; MARTINS, V.A.; TOMAZ, I.F. Análise da transmissão de preço e câmbio sobre os preços da farinha de trigo na cidade de São Paulo utilizando modelos de séries temporais. In: ENCONTRO DE ECONOMIA DA REGIÃO SUL - ANPEC-SUL, 9., Florianópolis, 2006. Anais... Florianópolis: ANPEC, 2006. 23 p. (CD).

MARTINES FILHO, J.G. Margens de Comercialização e causalidade de preços agrícolas. Piracicaba, 1988. 146 p. Dissertação (Mestrado em Economia Aplicada) - Escola Superior de Agricultura "Luiz de Queiroz", Universidade de São Paulo, Piracicaba, 1988.

MELO FILHO, G.A. de; OTSUBO, A.A. Estacionariedade e tendência dos preços de mandioca em Mato Grosso do Sul. Comunicado Técnico. Embrapa, Dourados, n.4, ago. 1999, p.1-3.

OTSUBO, A.A.; LORENZI, J.O. Cultivo de mandioca na região Centro-Sul do Brasil. Sistemas de produção 3, Dourados: Embrapa Agropecuária Oeste; Campinas: IAC; Cruz das Almas: Embrapa Mandioca e Fruticultura, 2002. 
SANTANA, F.S.; MELO, E.S.; CARDOSO, C.E.L; SOUZA, J.da S.; CALDAS, R.C. Variação de preços da raiz de mandioca no Estado da Bahia. In: CONGRESSO BRASILEIRO DE MANDIOCA, 11., Campo Grande, 2005. Anais... Campo Grande: Embrapa Agropecuária Oeste, 2005. 4p. (CD).

SECRETARIA DE ESTADO DA AGRICULTURA E DO ABASTECIMENTO DO PARANÁ - SEAB. Preços. Disponível em: <http://www.pr.gov.br/seab/>. Acesso em: 10 fev. 2006.

SOUZA, N. de J. de; STÜLP, V.J. Relações de trocas e causalidade de Granger entre preços pagos e recebidos pela agricultura brasileira, 1986/ 2004. Revista de Economia e Sociologia Rural, v. 43, n. 2, abr./jun. 2005. p. 267-285.

\begin{abstract}
This study aims to analyze the relations of causality and price transmission of the cassava root, the cassava starch, the cassava flour, the wheat, the wheat flour and the corn, in the State of Parana. The used data goes from January 1995 to December 2005 , with monthly regularity. It was applied the methodology of Granger causality to test the relations between the variables. The results showed long term relations between corn and cassava root prices, cassava starch and cassava flour prices, wheat and corn prices and wheat and wheat flour prices. It was emphasized the dependence of the cassava root prices in relation to corn, wheat, cassava starch and cassava flour prices and its little influence on these variables. It was also noted that cassava flour prices influenced the cassava starch prices, and the strong relation between the wheat prices and the wheat flour prices. The cassava flour and the wheat prices presented lesser dependence of the others variables. The paper concluded that there are important interrelations between the studied variables. The cassava root prices were the ones that depended most on the other variables in this study.
\end{abstract}

Key words: Granger causality, price transmission, starch. 